Rechtsmedizin 2020 • 30:153-160 https://doi.org/10.1007/s00194-020-00388-y Online publiziert: 21. April 2020

(c) Der/die Autor(en) 2020

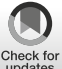

\author{
D. Brackrock · M. Dokter · C. Eckhoff · K.-P. Philipp · B. Bockholdt
}

Institut für Rechtsmedizin, Universitätsmedizin Greifswald, Greifswald, Deutschland

\title{
Gewalt gegen Frauen - eine Auswertung von sieben Jahren Gewaltopferambulanz am Institut für Rechtsmedizin Greifswald (2011-2017)
}

\section{Einleitung}

In der Vergangenheit hat sich die rechtsmedizinische körperliche Untersuchung lebender Personen zu einem elementaren Bestandteil der rechtsmedizinischen Routine entwickelt. Ziel der klinischen Rechtsmedizin ist es, Verletzungen, die durch fremde Gewalt verursacht wurden, umfassend und exakt zu dokumentieren, ggf. Spuren zu sichern und die Verletzungsmuster gutachterlich zu bewerten [10]. Nicht in jedem Fall erfolgt zeitnah eine rechtsmedizinische körperliche Untersuchung im Auftrag der Strafverfolgungsbehörden. Insbesondere in Fällen häuslicher Gewalt erstattet nur ein Bruchteil der Betroffenen unmittelbar eine Strafanzeige. Eine Studie des Kriminologischen Forschungsinstituts Niedersachsen aus dem Jahr 1992 ergab, dass lediglich $9 \%$ aller Fälle von häuslicher Gewalt durch die betroffenen Frauen zur Anzeige gebracht wurden [17]. Dies liegt u. a. darin begründet, dass Frauen den Kontakt zu den Ermittlungsbehörden aus Furcht vor einer möglichen Eskalation der Gewalt sowie einer Zerstörung der Familie mit ggf. einer Wegnahme der Kinder trotz wiederholter und langjähriger Gewalterfahrungen in der Partnerschaft vermeiden [13]. Selbst gegenüber ihren behandelnden Ärzten sprechen weniger als $10 \%$ der betroffenen Frauen ihre Gewalterfahrungen an, da sie sich „nicht trauen“ oder annehmen, dass die Proble- matik für den behandelnden Arzt nicht relevant ist [6].

Eine Studie der Europäischen Agentur für Grundrechte (FRA-Studie) aus dem Jahr 2014 wies für Deutschland im Vergleich mit anderen EU-Mitgliedsstaaten eine mittlere bis hohe Prävalenz von häuslicher Gewalt für Frauen aus [4]. Nach der Kriminalstatistik des Bundeskriminalamts waren 2015 von 127.457 Fällen erfasster Partnerschaftsgewalt $82 \%$ der Betroffenen weiblich [2]. Dies macht die dringende Notwendigkeit für niederschwellige Angebote deutlich, die den von Gewalt betroffenen Frauen die Möglichkeit geben, ihre Gewalterfahrungen in einem geschützten Rahmen zu offenbaren und ihre Verletzungen gerichtsfest dokumentieren zu lassen insbesondere dann, wenn keine Anzeige erstattet wurde und somit die Möglichkeit einer polizeilich angeordneten Dokumentation entfällt [20]. Zu diesem Zweck wurden in den letzten Jahren an den rechtsmedizinischen Instituten in Deutschland "Gewaltopferambulanzen“ etabliert. Diese Einrichtungen offerieren niederschwellig eine gerichtsfeste Verletzungsdokumentation.

Mit dem Inkrafttreten des Gewaltopferschutzgesetzes im Jahr 2002 wurden zunehmend mehr Gewaltopferambulanzen an den deutschen rechtsmedizinischen Instituten implementiert [5]. In Vorpommern wurde die Gewaltopferambulanz Ende des Jahres 2010 im Rahmen der Auftaktveranstaltung der „An- tigewaltwoche“ in der Universitäts- und Hansestadt Greifswald gegründet.

Eine rechtsmedizinische Verletzungsdokumentation erfasst entgegen einer klinischen Dokumentation Verletzungen unter forensischen Gesichtspunkten. Die rechtsmedizinischen Institute in Deutschland praktizieren im Rahmen der Gewaltopferversorgung ein insgesamt sehr ähnliches Vorgehen [1, 3]. So werden beispielsweise Verletzungen wahrgenommen und festgehalten, die möglicherweise unter klinischen Aspekten als nichtrelevant erscheinen, jedoch aus forensischer Sicht Hinweise auf den Tathergang liefern können. Eine Untersuchung aus Düsseldorf zeigte in diesem Zusammenhang, dass niedergelassene und klinisch tätige Ärzte Hilfsbedarf bei der erforderlichen Dokumentation haben [14]. Die angefertigte Dokumentation wird im Institut für Rechtsmedizin Greifswald zeitlich unbegrenzt aufbewahrt. Außerdem besteht für die Betroffenen die Möglichkeit, Spuren asservieren zu lassen. Bei Bedarf können Empfehlungen für weiterführende ärztliche Untersuchungen und Informationen zu Opferschutzinstitutionen und -angeboten gegeben werden. Die Untersuchung unterliegt der ärztlichen Schweigepflicht.

Die vorliegende Untersuchung soll zeigen, welchen Stellenwert Frauen im Untersuchungsgut der Gewaltopferambulanz Greifswald einnehmen, und Ansatzpunkte definieren, um die Er- 


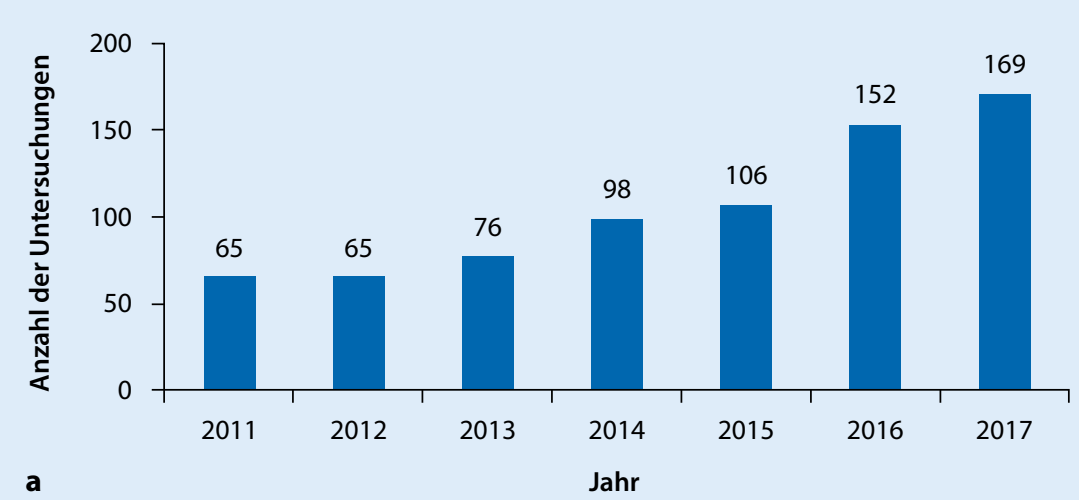

a

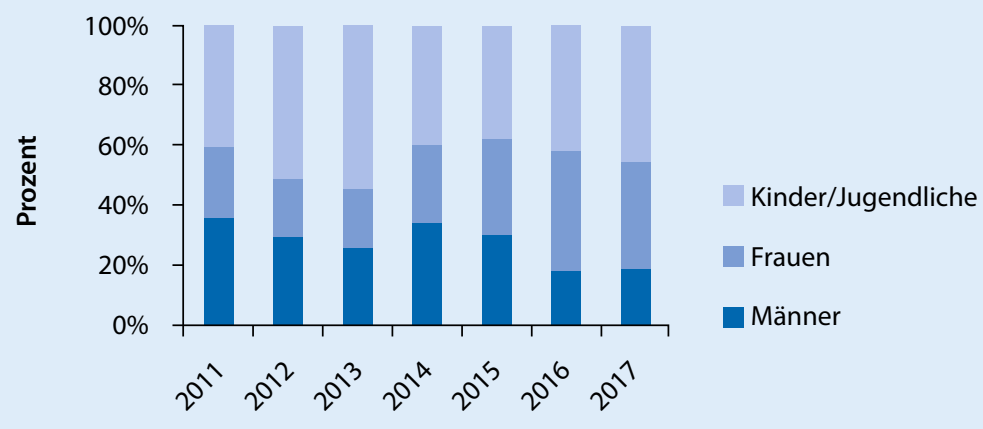

b

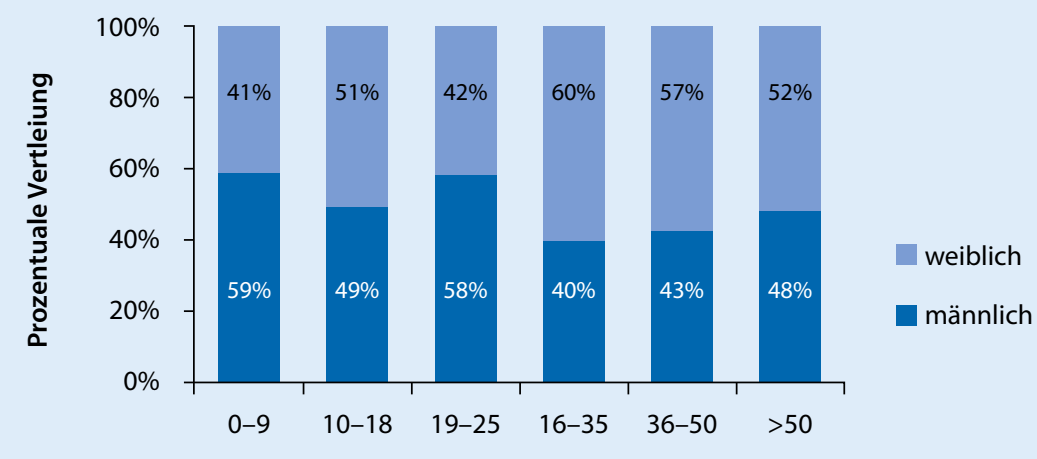

c

Alter (Jahre)

Abb. 1 A Entwicklung der Untersuchungszahlen sowie Alters- und Geschlechtsverteilung. a Zeitliche Entwicklung der Untersuchungszahlen, b prozentuale Verteilung der Untersuchungszahlen, c Altersund Geschlechtsverteilung

reichbarkeit gewaltbetroffener Frauen $\mathrm{zu}$ verbessern. Dazu wurde eine retrospektive Querschnittsstudie der in diesem Zusammenhang erfassten Daten aus den Jahren 2010-2017 aus dem Institut für Rechtsmedizin Greifswald (IREM) durchgeführt.

\section{Material und Methoden}

Grundlage der vorliegenden Untersuchung sind die im Rahmen der Gewaltopferambulanz des IREM Greifswald res wurden die Untersuchungsanlässe, Untersuchungsorte und die Zuweiser der Untersuchungen sowie die Art der Gewalteinwirkung betrachtet. Fälle, in denen einzelne Kriterien nicht dokumentiert waren, wurden für die entsprechende Auswertung ausgeschlossen. Die deskriptive Analyse der erhobenen Daten erfolgte mittels Pivot-Tabellen in Microsoft Excel (Microsoft Corporation, Redmond, WA, USA).

\section{Ergebnisse}

\section{Entwicklung der Untersuchungs- zahlen}

Insgesamt stellten sich im Untersuchungszeitraum 731 Personen in der Gewaltopferambulanz des IREM Greifswald vor, von denen 215 Personen Frauen ab dem 18. Lebensjahr waren. Im zeitlichen Verlauf konnte zwischen 2011 und 2017 ein deutlicher, kontinuierlicher Anstieg der Fallzahlen festgestellt werden. So wurden 2011 und 2012 je 65 Untersuchungen durchgeführt, während 2015 bereits 106 Untersuchungen stattfanden. Ein deutlicher Sprung in den Fallzahlen $(+43 \%)$ konnte 2016 mit insgesamt 152 Untersuchungen festgestellt werden (- Abb. 1a).

\section{Prozentuale Verteilung der Untersuchungszahlen}

Es wurde untersucht, wie sich die geschlechtsspezifische Entwicklung der Untersuchungszahlen im Zeitraum 20112017 darstellte. Zur Vollständigkeit wurden die Kinder und Jugendlichen bis zum 17. Lebensjahr, unabhängig vom Geschlecht, mitaufgenommen. Hier zeigte sich, dass die Zahl der untersuchten Kinder im Durchschnitt bei etwa $40 \%$ lag. Bei geschlechtsspezifischer Betrachtung der untersuchten Erwachsenen lässt sich feststellen, dass zunehmend mehr Frauen untersucht werden. So betrug der Anteil der Frauen zu Beginn der Etablierung der Gewaltopferambulanz (2011-2014) zwischen 40 und 44\%, während 2015 erstmalig mehr Frauen als Männer (52\% vs. $48 \%$ ) untersucht worden sind. Während der bisher höchste Anteil der untersuchten Frauen an der 
Gesamtzahl der untersuchten erwachsenen Personen im Jahr 2016 bei $70 \%$ lag, betrug dieser im Jahr $201765 \%$ (• Abb. 1b).

\section{Alters- und geschlechtsspezifische Verteilung}

Es wurde weiterhin untersucht, wie sich die alters- und geschlechtsspezifische Verteilung der untersuchten männlichen und weiblichen Personen in der Gewaltopferambulanz insgesamt darstellte. Hierzu wurden die Betroffenen in die Alterskategorien 0 bis 9 Jahre, 10 bis 18 Jahre, 19 bis 25 Jahre, 26 bis 35 Jahre, 36 bis 50 Jahre und $>50$ Jahre unterteilt. Hier war ein Überwiegen des Anteils erwachsener Frauen in den Altersgruppen 26 bis 35 Jahre ( $90 \%$ vs. $\sigma^{7}$ $40 \%$ ), 36 bis 50 Jahre (ㅇ $57 \%$ vs. $0^{7} 43 \%$ ) und bei den über 50 -jährigen ( $952 \%$ vs. $\sigma^{\top} 48 \%$ ) festzustellen. Ein höherer Männer- als Frauenanteil zeigte sich v.a. in der Gruppe der jungen Erwachsenen im Alter von 19 bis 25 Jahren (ㅇ $42 \%$ vs. O $58 \%$ ) (- Abb. 1c).

\section{Zuweiser}

Um ein besseres Verständnis hinsichtlich der Zuweisungswege insbesondere gewaltbetroffener Frauen zu erlangen, wurden die Fälle von untersuchten Frauen $(n=215)$ in die Kategorien "Selbstvorstellung“ und „Klinik“" unterteilt. In die Kategorie „Klinik“ wurden dabei alle Fälle aufgenommen, bei denen die Betroffene konsiliarisch während eines stationären Aufenthalts in einer Klinik des Einzugsgebietes der Rechtsmedizin Greifswald, auf Anforderung der behandelnden Ärzte, durch einen Rechtsmediziner untersucht wurden. Dieser Anteil schwankt im Verlauf der Jahre etwas und lag zuletzt zwischen 10 und $20 \%$. Der Anteil der Frauen, die sich auf eigenen Wunsch bzw. selbstständig zur Untersuchung vorstellten, lag im gesamten Untersuchungszeitraum auf einem sehr hohen Niveau zwischen $67 \%$ (2012) und 91\% (2017) (- Abb. 2a).

Bei allen Frauen, die sich in der Gewaltopferambulanz selbstständig vorstellten, wurden außerdem Informationen $\mathrm{zu}$ den vermittelnden Institutio-

Rechtsmedizin 2020 · 30:153-160 https://doi.org/10.1007/s00194-020-00388-y

(c) Der/die Autor(en) 2020

D. Brackrock · M. Dokter · C. Eckhoff · K.-P. Philipp · B. Bockholdt

\section{Gewalt gegen Frauen - eine Auswertung von sieben Jahren Gewaltopferambulanz am Institut für Rechtsmedizin Greifswald (2011-2017)}

\section{Zusammenfassung}

Die körperliche Untersuchung gewaltbetroffener, lebender Personen, aber auch in Fällen von Selbstbeschädigung, gehört zur täglichen Routine der rechtsmedizinischen Institute. Gleichwohl sind auch Ärzte anderer Fachrichtungen mit Opfern von fremder Gewalt konfrontiert. Die vorliegende Studie soll die Bedeutung einer gerichtsfesten Befunddokumentation bei Betroffenen häuslicher Gewalt außerhalb eines Strafverfahrens unterstreichen. In einer retrospektiven Querschnittsstudie wurden die Daten aus der rechtsmedizinischen Gewaltopferambulanz des Instituts für Rechtsmedizin Greifswald der Jahre 2011-2017 systematisch unter- sucht. Die Untersuchung ergab u. a. einen steigenden Anteil weiblicher Betroffener im Untersuchungsgut und als häufigsten Untersuchungsanlass den Verdacht auf häusliche Gewalt. Durch die jährlich steigenden Fallzahlen wird die Bedeutung der rechtsmedizinischen Gewaltopferambulanz für die umfassende Versorgung von Betroffenen von (häuslicher) Gewalt deutlich.

Schlüsselwörter

Misshandlung · Häusliche Gewalt · Beweisführung · Klinische Rechtsmedizin . Befunddokumentation

\section{Violence against women-an evaluation of 7 years of the outpatient clinic for victims of violence at the Greifswald Institute for Forensic Medicine (2011-2017)}

\section{Abstract}

The physical examination of living victims of violence and also in cases of self-harming behavior is part of the daily routine at forensic institutes. Additionally, physicians in other disciplines are also confronted with victims of violence. The present study emphasizes the importance of a legally valid documentation of findings in cases of domestic violence outside of criminal proceedings. In a retrospective cross-sectional study, data collected from 2011-2017 by the forensic outpatient clinic for victims of violence of the Greifswald Institute for Forensic Medicine were systematically examined. The investigation showed an increasing proportion of female patients in the investigation material and suspected domestic violence as the most common reason for investigations. The study confirmed the importance of the forensic service for victims of violence for the comprehensive care for victims of (domestic) abuse by the annually increasing numbers of cases.

\section{Keywords}

Violence mistreatmanet - Domestic abuse · Proof · Forensic medicine · Trauma documentation nen erfasst. Zur Auswertung wurden hier die Gruppen „Interventionsstellen/Frauenhäuser“, „Krankenhäuser“, „niedergelassene Ärzte“, „Polizei“ und "Sonstige“ (Vermittlung durch Familienhelfer, Anwalt, Betreuer, Psychologe u. Ä.) gebildet. Besonders häufig wurden die Frauen über Interventionsstellen (72 Betroffene) an die Gewaltopferambulanz vermittelt. Durch Kliniken wurden 24 Betroffene vermittelt. Mit insgesamt 14 Betroffenen, die eine Empfehlung durch die Polizei erhielten, bildete dies die dritthäufigste Kategorie. Nur 9 Be- troffene erhielten Informationsmaterial zur Gewaltopferambulanz durch niedergelassene Ärzte bzw. den Hausarzt (-Abb. 2b).

\section{Untersuchungsorte}

Es wurde weiterhin ausgewertet, wo die Untersuchung der betroffenen Frauen $(n=211)$ stattfand. Die Untersuchungsorte wurden dabei in die Kategorien „Klinik“, „Institut“ (IREM), „Interventions- und Beratungsstelle“, „Frauenhaus“, „Häuslichkeit“ und „Sonstige“ 

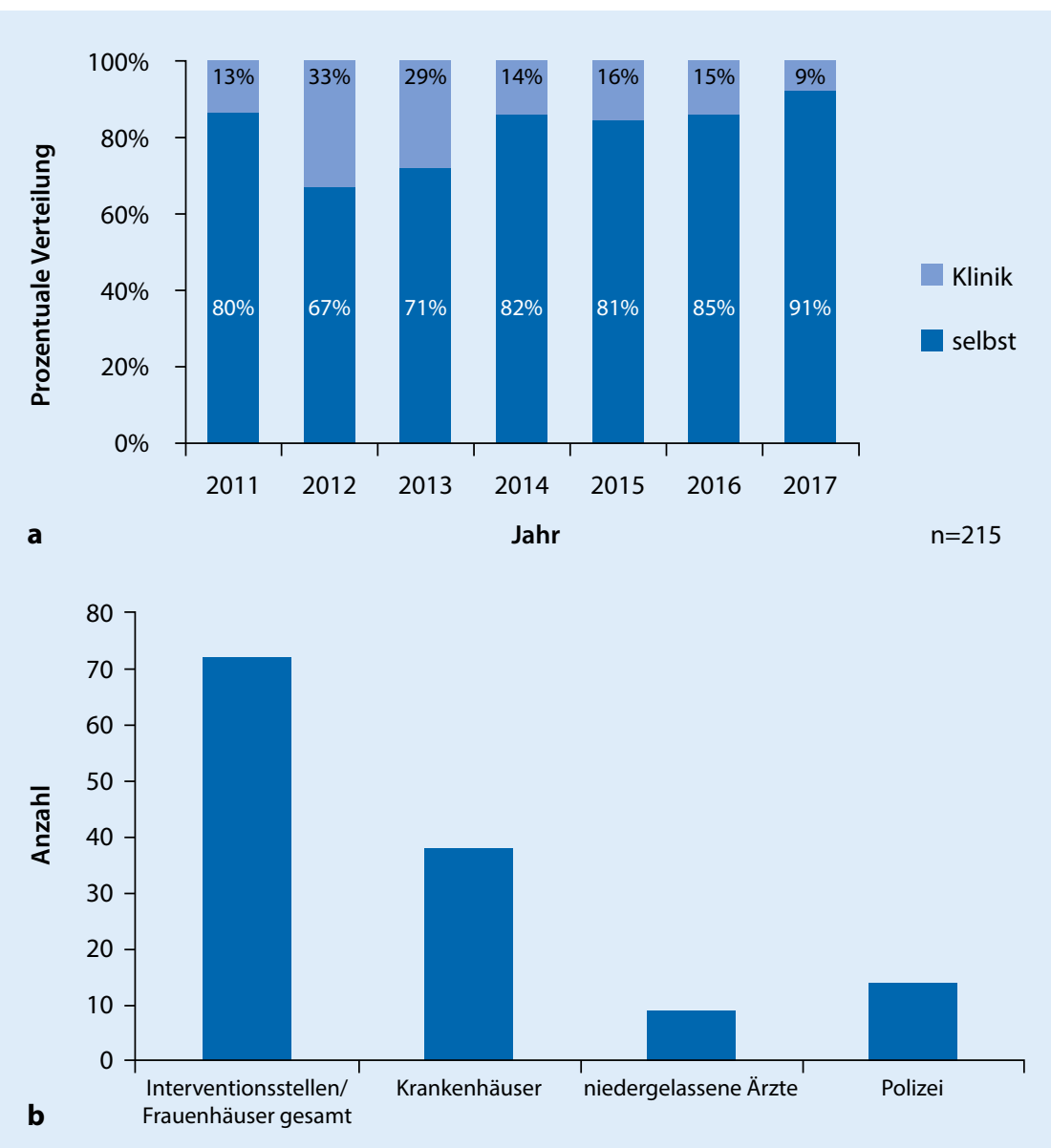

Abb. 2 \ Häufigkeitsverteilung von Zuweisern und Vermittlern. a Zuweiser untersuchte Frauen, bVermittler bei Selbstvorstellung

(u. a. Praxis, Gesundheitsamt) eingeteilt. Bei der Auswertung zeigte sich, dass die betroffenen Frauen etwa gleich häufig in einem der umliegenden Krankenhäuser des Einzugsgebietes des IREM (30\%, 63 Betroffene) oder im IREM selbst (31\%, 65 Betroffene) untersucht wurden.Häufig wurden für die Untersuchung auch Räumlichkeiten von Interventionsund Beratungsstellen (27\%, 58 Betroffene) zur Verfügung gestellt. Darüber hinaus fanden Untersuchungen weiblicher Gewaltopfer in Frauenhäusern (4\%, 8 Betroffene) statt (• Abb. 3 ).

\section{Untersuchungsanlässe}

Bei der Auswertung der Untersuchungsanlässe in Fällen, bei denen Frauen untersucht wurden $(n=215)$, wurde unterschieden, ob der Vorfall im häuslichen Rahmen stattfand (häusliche Gewalt), oder ob eine fremde Person, die nicht zum häuslichen Umfeld gehört, als Verursacher genannt wurde. Entsprechend diesem Ansatz wurden 4 Hauptgruppen gebildet: 1. „V.a. häusliche Gewalt“, 2. „V.a. Rohheitsdelikt“, 3. „V.a. sexuelle Gewalt" und 4. „Sonstige“ (u. a. Verdacht fall, V.a. Unfall). Im gesamten Untersuchungszeitraum überwog der Anteil von Untersuchungen bei Frauen wegen des Verdachts häuslicher Gewalt (>60\%). Ab dem Jahr 2015 und besonders im Jahr 2016 konnte eine deutliche Zunahme der Fälle in dieser Gruppe festgestellt werden (2016: 45 Betroffene, 79\%). Es zeigte sich, dass die Zunahme der Vorstellung bei Verdacht auf häusliche Gewalt proportional mit der Zunahme der Untersuchungszahlen weiblicher erwachsener Personen insgesamt erfolgte. Der Untersuchungsanlass „V.a. häusliche Gewalt" stellt somit den häufigsten und damit bedeutsamsten Anlass bei der Unauf Selbstbeibringung, V.a. Raubüber- tersuchung von erwachsenen Frauen dar. Der zweithäufigste Untersuchungsanlass bei Frauen war der Verdacht auf ein Rohheitsdelikt. Dieser war im Untersuchungszeitraum sehr variabel (bis $46 \%$ im Jahr 2012), jedoch war in den Jahren 2016 und $2017 \mathrm{zu}$ beobachten, dass sich dieser Vorstellungsgrund auf einen Anteil zwischen 7 und 15\% reduzierte. Relativ selten erfolgten Untersuchungen wegen des Verdachts auf sexuelle Gewalt (2-13\%) (• Abb. 4a, b).

\section{Art der erfahrenen Gewalt- einwirkung}

Bei jeder Untersuchung wurde erfasst, durch welche Art der Gewalteinwirkung die festgestellten Verletzungen verursacht wurden. Es wurden die Kategorien „stumpfe Gewalteinwirkung“ (Schürfungen, Unterblutungen, Knochenbrüche u. Ä.), „scharfe Gewalteinwirkung“ (Stich- und Schnittverletzungen), „Halskompression“, „keine relevanten Verletzungen" und die Kategorie "Sonstige“ (u.a. Schuss, Verätzung, thermische Verletzungen) festgelegt. Wenn bei einer Untersuchung mehrere Verletzungsarten vorlagen, erfolgte eine Mehrfachzuordnung jeweils $\mathrm{zu}$ den oben genannten Gruppen. Als Grundgesamtheit wurde somit die Gesamtzahl der festgestellten Gewaltarten angenommen. Die Analyse der Art der eingewirkten Gewalt im Rahmen der Untersuchung von Frauen $(n=205)$ zeigte, dass Verletzungen durch stumpfe Gewalteinwirkung mit $84 \%$ den weitaus größten Anteil darstellten. Verletzungen nach Halskompression bildeten mit $8 \%$ der festgestellten Gewaltarten die zweithäufigste Gruppe. Bei $4 \%$ der untersuchten betroffenen Frauen konnten keine relevanten Verletzungen festgestellt werden, d.h., die Betroffenen wiesen keine oder ausschließlich für den Vorfall nichtrelevante Bagatellverletzungen auf (• Abb. 5).

\section{Veranstaltungen zur Bekanntgabe}

In den Jahren 2011-2017 wurden durch die Ärzte des IREM Greifswald insgesamt 132 Veranstaltungen (in der Regel Vorträge) durchgeführt, bei denen das Angebot zur niederschwelligen rechtsme- 


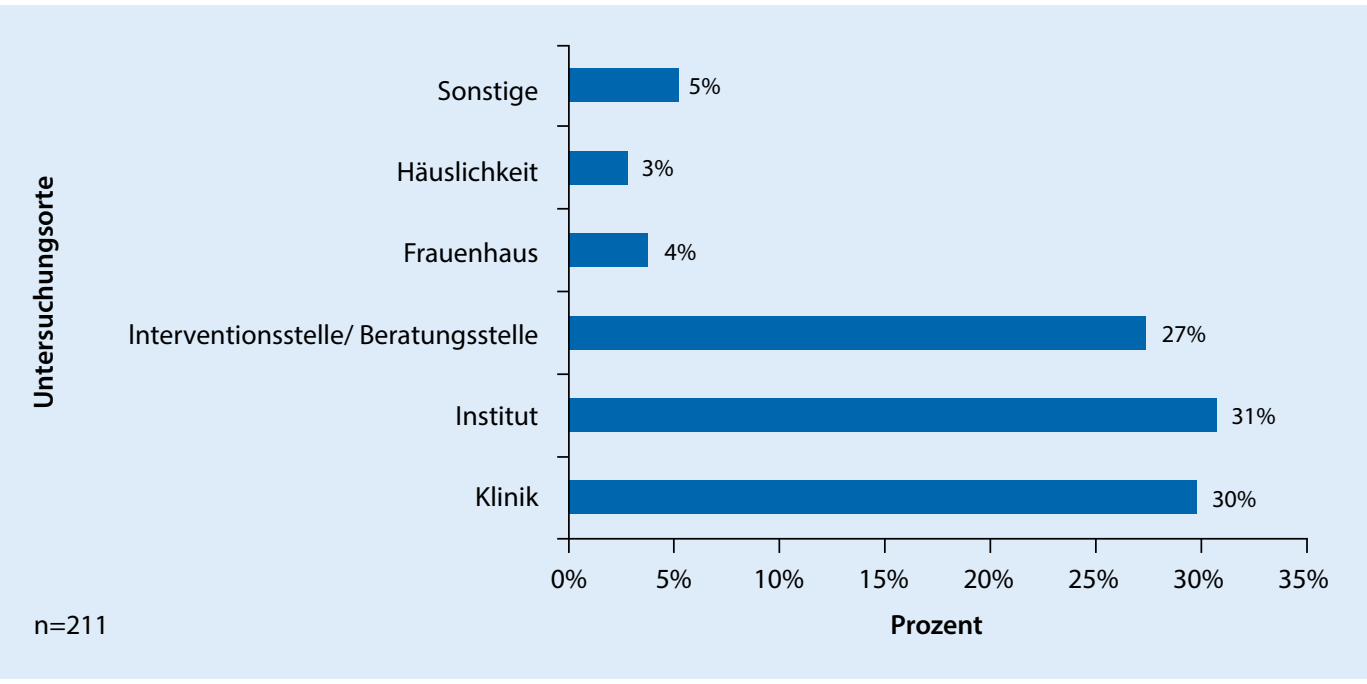

Abb. $3<$ Darstellung der Untersuchungsorte

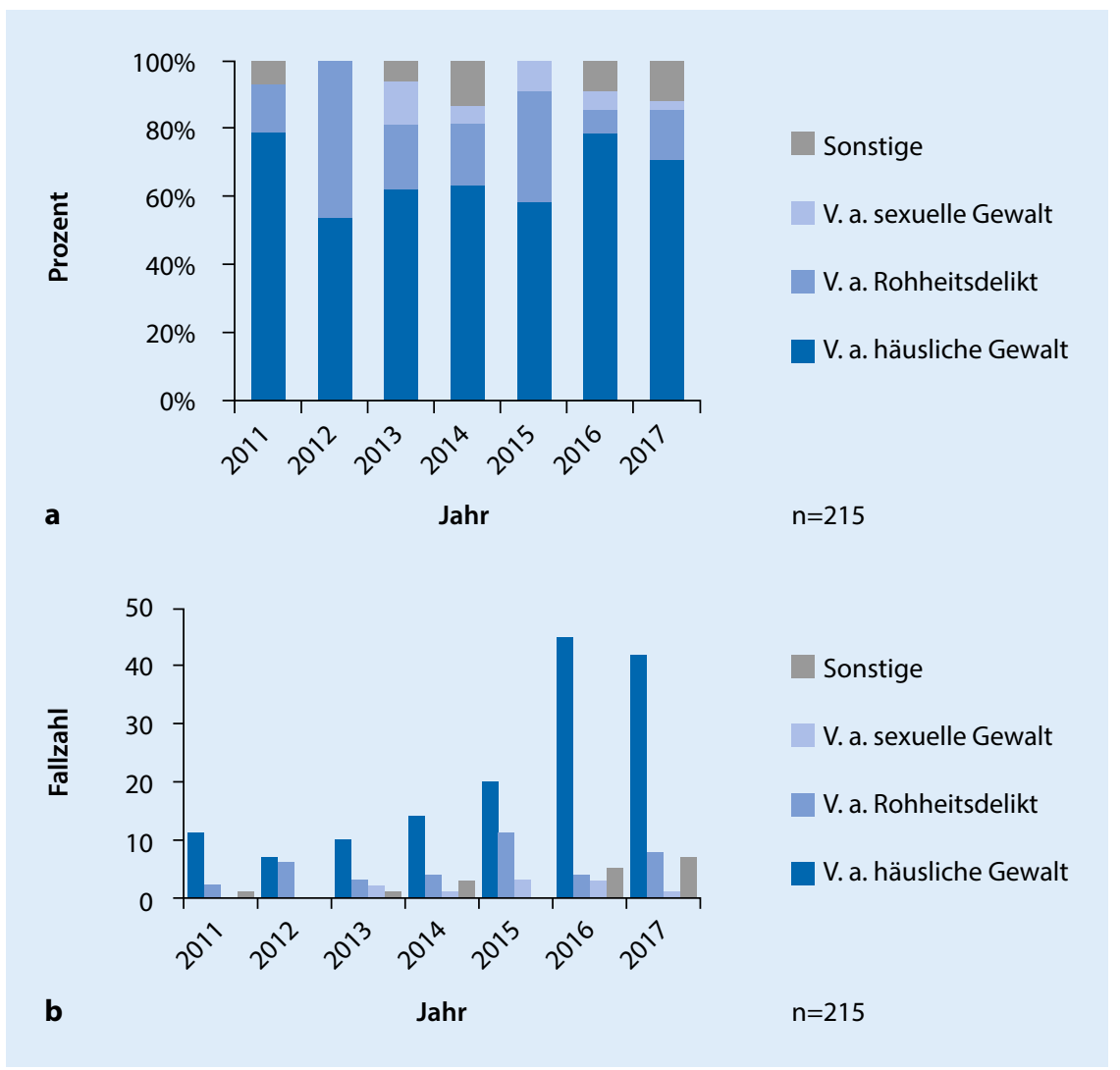

Abb. 4 A Auswertung der Untersuchungsanlässe und Gewaltarten. a Untersuchungsanlässe Frauen, b Entwicklung der Untersuchungsanlässe bei Frauen

dizinischen Untersuchung im Rahmen der Gewaltopferambulanz für Betroffene fremder Gewalt bekannt gegeben und über die Notwendigkeit dieser Untersuchungen informiert wurde. Am häufigsten wurden Ärzte und anderes medizinisches Personal $(n=44)$, gefolgt von Polizei und Justiz $(n=29)$ sowie Hilfsein-

\section{Diskussion}

Im Untersuchungszeitraum zwischen 2011 und 2017 konnte ein kontinuierlicher Anstieg der Untersuchungszahlen, insbesondere der der weiblichen Betroffenen, beobachtet werden. $\mathrm{Ab}$ einem Alter >25 Jahren waren Frauen besonders häufig von Gewalt betroffen. Aus Sicht der Autoren markiert sich ab dieser Altersgruppe insbesondere die Betroffenheit von häuslicher Gewalt. Diese Zahlen belegen, dass die Gewaltopferambulanz am IREM Greifswald zunehmend die Zielgruppe (betroffene Frauen häuslicher Gewalt) erreicht. Ähnliche Ergebnisse konnten bereits andere rechtsmedizinische Institute erheben: Bei einer retrospektiven Auswertung der Daten der Untersuchungsstelle des Instituts für Rechtsmedizin Hamburg waren $70,1 \%$ der untersuchten Frauen zwischen 2003 und 2009 Opfer von Gewalt in (Ex-)Partnerschaften und sexueller Gewalt geworden [4]. Beachtenswert ist dieser Umstand v. a. deshalb, da gemäß einer repräsentativen Umfrage jede zweite von (häuslicher) Gewalt betroffene Frau in der Regel nicht über ihre Erfahrungen spricht [17]. Die Niederschwelligkeit der Gewaltopferambulanz kann hier das entscheidende Kriterium sein, dass diese Frauen sich dennoch in der Gewaltopferambulanz vorstellen.

Untersuchungen von Frauen wegen des Verdachts auf sexuelle Gewalt oder eines Rohheitsdelikts außerhalb häusli- 


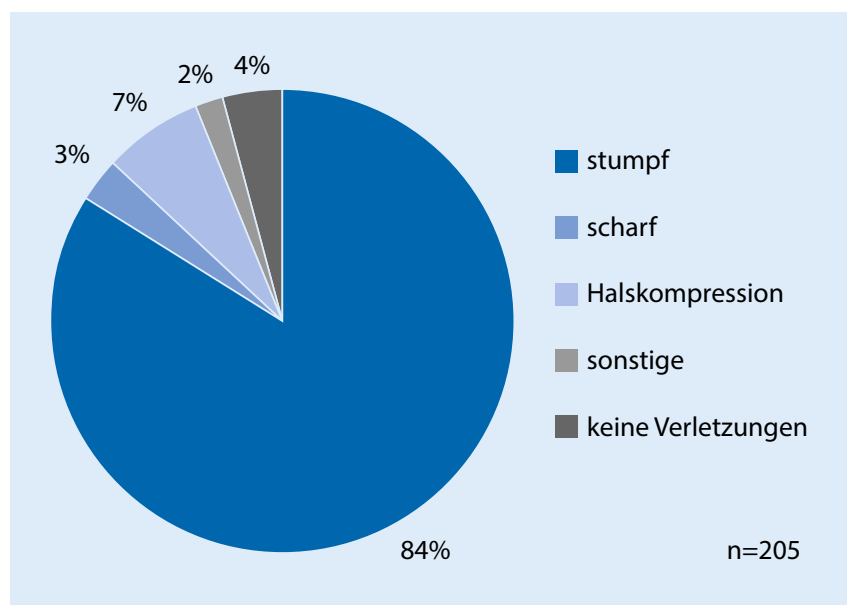

Abb. $5<$ Darstellung der Art der Gewalteinwirkung

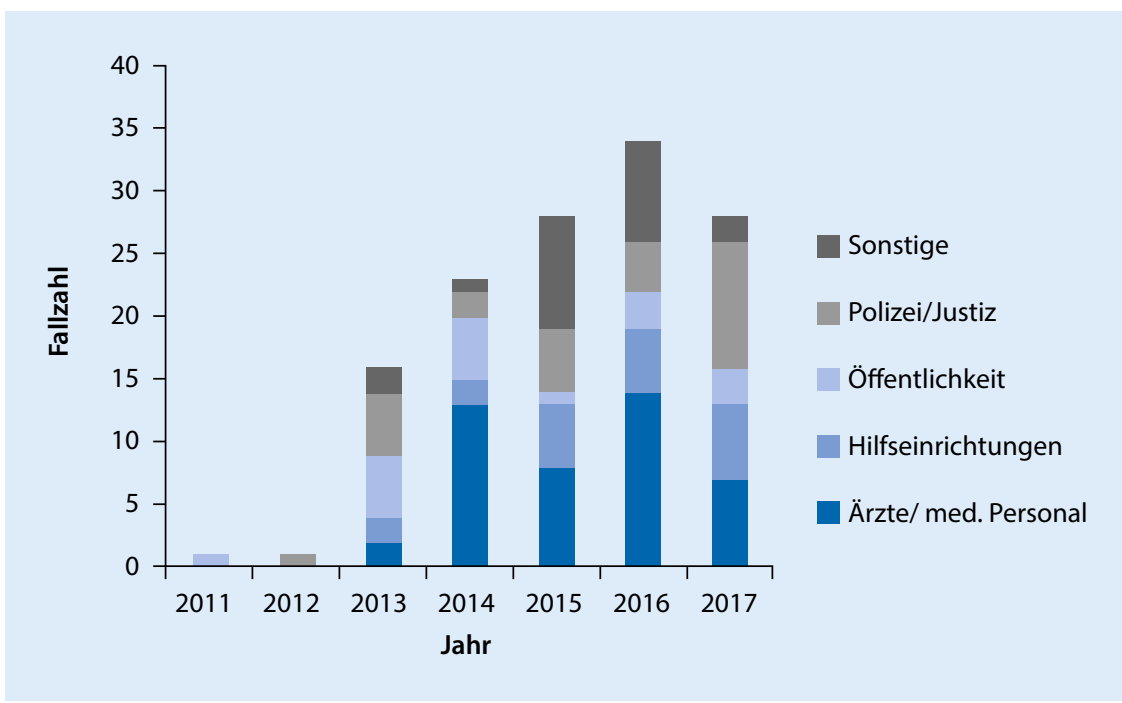

Abb. 6 \ Veranstaltungen zur Bekanntgabe der Gewaltopferambulanz

cher Gewalt waren deutlich seltener in unserem Untersuchungsgut.

Unsere retrospektive Analyse ergab außerdem, dass die vorherrschenden Verletzungen der weiblichen Gewaltopfer sich in unserem Untersuchungsgut überwiegend auf stumpfe Gewalteinwirkung zurückführen ließen, was sich mit den Ergebnissen anderer Untersuchungen deckt. Wischmann et al. stellten in $79,9 \%$ der Fälle als Ursache der Verletzungen stumpfe ungeformte Gewalteinwirkung fest [19]. Bei der genaueren Betrachtung der Verletzungsformen fanden Jungbluth et al. vorwiegend Hämatome bei den Betroffenen [7].

Die betroffenen Frauen wurden überwiegend im IREM oder in einer Klinik bzw. einer Beratungs- bzw. Interventionsstelle im Einzugsbereich untersucht.
Anders als in Großstädten, wo eine fest installierte Gewaltopferambulanz ausreichend ist, erfordert dies in ländlichen Gegenden, wie dem östlichen Mecklenburg-Vorpommern (Landkreise Vorpommern-Greifswald, VorpommernRügen, Mecklenburgische Seenplatte) eine mobile 24-h-Rufbereitschaft der Rechtsmedizin auch für die Gewaltopferambulanz [15]. Dies ist für die Rechtsmediziner jedoch mit einem hohen Fahr- und Zeitaufwand verbunden. Dennoch kann aus Sicht der Autoren nur so gewährleistet werden, dass die Betroffenen zeitnah untersucht werden und das Angebot seine Niederschwelligkeit behält. Zudem können auf diesem Weg auch eine zum Vorfallszeitpunkt möglichst zeitnahe Untersuchung und Befunddokumentation für die betroffenen Frauen ermöglicht werden, was beispielsweise in der Dokumentation von Petechien in Fällen von Halskompression essenziell ist [16].

Ein weiterer wesentlicher Faktor, der die Akzeptanz bei den Betroffenen verstärkt, ist, dass die Untersuchung, Dokumentation und Befundarchivierung sowohl in Greifswald als auch vielen weiteren Instituten in Deutschland, Österreich und der Schweiz für die Betroffenen kostenfrei sind [5]. Die Gegenfinanzierung der Gewaltopferambulanzen erfolgt bundesweit in unterschiedlichen Modellen [13]. Diese Möglichkeiten sind in der Regel nicht kostendeckend; hier muss offenbar regional für eine zukunftsträchtige Finanzierung gesorgt werden [9].

Es zeigte sich in unserer Auswertung, dass die weiblichen Opfer sich in $80-90 \%$ der Fälle selbst, also auf eigene Initiative, vorstellten. Dies deckt sich mit einer Umfrage von Gahr et al. unter den rechtsmedizinischen Instituten in Deutschland, Österreich und der Schweiz, deren Auswertungen ergaben, dass sich durchschnittlich $86 \%$ der Betroffenen selbst in den Gewaltopferambulanzen vorstellen [5]. Ein Grund hierfür kann neben den bereits oben genannten Faktoren (kostenlose und „VorOrt-Untersuchung") auch wiederum die Niederschwelligkeit der Gewaltopferambulanz sein, die es den Frauen erlaubt, ihre Verletzungen in einem geschützten Rahmen dokumentieren zu lassen.

Es ist für die Frage der Erreichbarkeit von hoher Relevanz zu wissen, wer die Betroffenen zur Untersuchung vermittelte. Unsere Analyse ergab, dass ein großer Teil der Selbstvorstellungen durch Interventionsstellen gegen Gewalt, Frauenhäuser oder andere Hilfseinrichtungen an die Gewaltopferambulanz unseres Instituts vermittelt wurde. Dies zeigt, dass die Akzeptanz für das Angebot der Gewaltopferambulanz auch bei den Opferschutzorganisationen gestiegen ist. Sofern die Betroffenen sich noch nicht bei Opferschutzorganisationen vorgestellt haben, bietet sich auch im Rahmen der rechtsmedizinischen Befunddokumentation die Möglichkeit, Opfer von Gewalt über derartige Hilfseinrichtungen $\mathrm{zu}$ informieren und den Kontakt zu vermitteln. 
Ein weiterer Weg der Vermittlung erfolgte durch ärztliche Kollegen in den umliegenden Kliniken. Dies belegt aus unserer Sicht die Bedeutung einer guten Vernetzung mit den ansässigen Kliniken im Einzugsgebiet. Ärzte stellen in dieser Hinsicht Vertrauenspersonen für Betroffene häuslicher Gewalt dar. Das unterstreichen Studien, die ergaben, dass v. a. Allgemeinmediziner [8], die Notaufnahmen [11] aber auch gynäkologische, pädiatrische oder unfallchirurgische Kliniken aufgesucht werden [9]. Sie nehmen somit eine Schlüsselrolle bei der Versorgung von Betroffenen häuslicher Gewalt ein.

Ansatzpunkte für eine Sensibilisierung der ärztlichen Kollegen bieten sowohl Schulungen durch die rechtsmedizinischen Institute als auch neue Konzepte für den Studentenunterricht. Beispielhaft wurde für den Modellstudiengang Medizin der Charité Berlin ein Konzept entwickelt, das Medizinstudenten im Blockunterricht die Gesprächsführung bei häuslicher Gewalt und in einer weiteren Lehrveranstaltung die Dokumentation und forensische Spurensicherung lehrt [18]. Die in den Jahren 2011-2017 durch uns durchgeführten Schulungen von Ärzten und medizinischem Personal, der Polizei und Justiz sowie Hilfseinrichtungen und der Öffentlichkeit zur Gewaltopferambulanz, aber auch zu der Erkennbarkeit von fremder Gewalt allgemein, haben aus Sicht der Autoren wesentlich zu dem bereits aufgezeigten Anstieg der Untersuchungszahlen beigetragen. Da bereits gezeigt werden konnte, dass rechtsmedizinische Expertise in der Regel durch klinische Kollegen nur in Anspruch genommen wird, wenn auch für den rechtsmedizinischen Laien erkennbare Verletzungen vorliegen [12], tragen die genannten Schulungen auch zur besseren Versorgung der Gewaltbetroffenen bei. Insbesondere auch Schulungen der Schutzpolizei sollten aus unserer Sicht nicht vernachlässigt werden, da unserer Erfahrung nach in vielen Fällen häuslicher Gewalt bereits (wiederholte) Polizeieinsätze stattgefunden hatten, eine rechtsmedizinische körperliche Untersuchung nach $\$ 81 \mathrm{c}$ StPO demgegenüber aber nur sehr selten angeordnet wird und die betroffenen Frauen auch nicht in jedem Fall anderweitig ärztlich vorstellig werden. In diesem Zusammenhang könnte auch die Schutzpolizei ein (weiterer) wichtiger Vermittler für die Gewaltopferambulanz werden.

Die oben genannten Punkte machen deutlich, dass die Hilfe für betroffenen Frauen multifaktoriell aufgebaut werden muss, da jede Profession einen anderen Versorgungsschwerpunkt hat und erst durch eine entsprechende Vernetzung eine umfassende Versorgung bei häuslicher Gewalt gewährleisten kann. Die Gewaltopferambulanzen der rechtsmedizinischen Institute spielen hier eine wichtige Rolle.

\section{Fazit für die Praxis}

- Die rechtsmedizinische körperliche Untersuchung im Rahmen einer Gewaltopferambulanz ist ein notwendiges und nützliches Angebot, das (weiblichen) Betroffenen von Gewalt die Möglichkeit bietet, ihre Verletzungen in einem geschützten Rahmen gerichtsfest dokumentieren zu lassen.

- Die rechtsmedizinischen Gewaltopferambulanzen haben eine hohe Bedeutung bei der umfassenden Versorgung von Betroffenen (häuslicher) Gewalt.

- Schulungen von Ärzten und medizinischem Personal, der Polizei und Justiz sowie Hilfseinrichtungen und auch der Öffentlichkeit sind sinnvoll und wirkungsvoll, um Betroffene zu erkennen und zu erreichen.

- Eine sinnvolle Lösung zur Finanzierung dieser Angebote muss dringend gefunden werden, um den rechtsmedizinischen Instituten auch weiterhin die Möglichkeit zu geben, dieses Angebot aufrechtzuerhalten und weiter ausbauen zu können.

- In Mecklenburg-Vorpommern muss die rechtsmedizinische Gewaltopferambulanz mobil in der Fläche angeboten werden.

\section{Korrespondenzadresse}

\section{Dr. med. M. Dokter}

Institut für Rechtsmedizin, Universitätsmedizin Greifswald

Kuhstraße 30, 17489 Greifswald, Deutschland martin.dokter@med.uni-greifswald.de

Danksagung. Die Autoren danken Frau K. Runge und Frau S. Heise für die Unterstützung bei der Datenerhebung.

Funding. Open Access funding provided by Projekt DEAL.

\section{Einhaltung ethischer Richtlinien}

Interessenkonflikt. D. Brackrock, M. Dokter, C. Eckhoff, K.-P. Philipp und B. Bockholdt geben an, dass kein Interessenkonflikt besteht.

Für diesen Beitrag wurden von den Autoren keine Studien an Menschen oder Tieren durchgeführt. Für die aufgeführten Studien gelten die jeweils dort angegebenen ethischen Richtlinien.

Open Access Dieser Artikel wird unter der Creative Commons Namensnennung 4.0 International Lizenz veröffentlicht, welche die Nutzung, Vervielfältigung Bearbeitung, Verbreitung und Wiedergabe in jeglichem Medium und Format erlaubt, sofern Sie den/die ursprünglichen Autor(en) und die Quelle ordnungsgemäß nennen, einen Link zur Creative Commons Lizenz beifügen und angeben, ob Änderungen vorgenommen wurden

Die in diesem Artikel enthaltenen Bilder und sonstiges Drittmaterial unterliegen ebenfalls der genannten Creative Commons Lizenz, sofern sich aus der Abbildungslegende nichts anderes ergibt. Sofern das betreffende Material nicht unter der genannten Creative Commons Lizenz steht und die betreffende Handlung nicht nach gesetzlichen Vorschriften erlaubt ist, ist für die oben aufgeführten Weiterverwendungen des Materials die Einwilligung des jeweiligen Rechteinhabers einzuholen.

Weitere Details zur Lizenz entnehmen Sie bitte der Lizenzinformation auf http://creativecommons.org/ licenses/by/4.0/deed.de.

\section{Literatur}

1. Banaschak S, Gerlach K, Seifert D et al (2014) Forensisch-medizinische Untersuchung von Gewaltopfern. Rechtsmedizin 24:405-411

2. Bundeskriminalamt (2015) Partnerschaftsgewalt Kriminalstatistische Auswertung - Berichtsjahr 2015. In: Bundeskriminalamt (Hrsg) IZ33. Bundeskriminalamt, Wiesbaden

3. Feld K, Schulte C, Bogus M et al (2019) Anonyme Spurensicherung nach Sexualstraftaten in Köln und Umgebung - Erfahrungen aus mehr als 7 Jahren aus rechtsmedizinischer Perspektive. Rechtsmedizin 29:484-490

4. FRA European Union Agency for Fundamental Rights (2014) Violence against women: an EU-wide 
survey. Publications Office of the European Union, Luxembourg

5. Gahr B, Graß H, Ritz-Timme S et al (2012) Klinisch-rechtsmedizinische Kompetenz in der Gewaltversorgung. Rechtsmedizin 22:379-384

6. Jundt K, Friese K (2009) Gewalt an Frauen. Gynäkologe 42:723-727

7. Jungbluth P, Grassmann J-P, Wild $M$ et al (2012) Gewaltopferversorgung in der Notfallambulanz. Rechtsmedizin 22:163-168

8. Mark H (2000) Häusliche Gewalt gegen Frauen aus der Sicht niedergelassener Ärztinnen und Ärzte: Ergebnisse einer Befragung in den Berliner Bezirken Hohenschönhausen und Lichtenberg. Zeitschrift Für Gesundheitswissenschaften 8:332

9. Mützel E, Helmreich C, Schick Setal (2014) Klinischforensische Versorgung von Gewaltopfern in Bayern. Rechtsmedizin 24:200-207

10. Pollak S (2004) Clinical forensic medicine and its main fields of activity from the foundation of the German Society of Legal Medicine until today. Forensic Sci Int 144:269-283

11. Poloczek S, Schmitt TK (2002) Häusliche Gewalt - Eine Herausforderung für die Notfallmedizin. Notfall Rettungsmed 5:498-504

12. Seifert D, Heinemann A, Anders S et al (2006) Vergleichende klinisch-rechtsmedizinische Analyse von Verletzungsmustern. Rechtsmedizin 16:205-212

13. Seifert D, Heinemann A, Püschel K (2006) Frauen und Kinder als Opfer häuslicher Gewalt. Dtsch Arztebl 103(33):2168-2173

14. Siegel M, Gahr B, Tank A et al (2017) V35 iGOBSIS-live: Brauchen Ärztinnen und Ärzte wirklich Unterstützung in der Gewaltopferversorgung? Vortrag aufder 26. Frühjahrstagung der Deutschen Gesellschaft für Rechtsmedizin (Region Süd) in Verbindung mit dem 47. Treffen der Oberrheinischen Rechtsmediziner. Rechtsmedizin 27:475-490

15. Stanislawski N, Philipp K-P, Bockholdt B (2014) Untersuchungsstelle für Gewaltopfer am Institut für Rechtsmedizin der Universitätsmedizin Greifswald. Rechtsmedizin 24:258-262

16. Strauch H, Lignitz E, Geserick G (1990) Obstruktive Asphyxie (Würgen, Drosseln) mit Überleben. In: Brinkmann B, Püschel K (Hrsg) Ersticken Fortschritte in der Beweisführung Festschrift für Werner Janssen. Springer, Berlin, Heidelberg, S248-255

17. Wetzels P, Pfeiffer C (1995) Sexuelle Gewalt gegen Frauen im öffentlichen und privaten Raum Ergebnisse der KFN-Opferbefragung. In: Kriminologisches Forschungsinstitut Niedersachsen (Hrsg) Opferbefragung. KFN, Hannover

18. Wieners K, Winterholler M(2016) Häusliche und sexuelle Gewalt gegen Frauen. Bundesgesundheitsblatt Gesundheitsforschung Gesundheitsschutz 59:73-80

19. Wischmann $M$, Heinemann $A$, Püschel $K$ et al (2013) Frauen als Opfer von Gewalt. Rechtsmedizin 23:466-471

20. Yen K (2013) Aufgaben und Nutzen klinischforensischer Ambulanzen. In: Grassberger M, Türk E, Yen K (Hrsg) Klinisch-forensische Medizin. Springer, Berlin, Heidelberg, S 100-104
Konrad, N., Huchzermeier, C., Rasch, W.

\section{Forensische Psychiatrie und Psychotherapie}

Rechtsgrundlagen, Begutachtung und Praxis

Stuttgart: Kohlhammer Verlag 2019, 5 (erweitert und überarbeitet), 582 S., (ISBN: 978-3170336421), 148 EUR

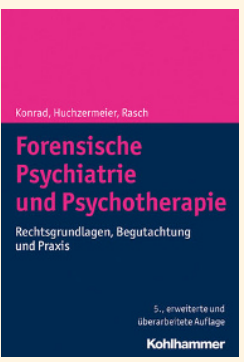

Die 5. Auflage dieses Hand- und Lehrbuchs zeigt ein gründlich überarbeitetes, didaktisch gut aufbereitetes Kompendium mit strafrechtlichem Schwerpunkt. In den erheblich erweiterten zentralen Kapiteln (Psychiatrische Grundlagen) stützt es sich als erstes deutschsprachiges Forensikfachbuch auf die aktualisierte Internationale Klassifikation (ICD-11) und erläutert differenziert deren Auswirkungen auf die Begutachtungspraxis. Dass die Anwendung der ICD-11 die individualisierende klinische Analyse nicht erübrigt, benennen die Autoren mit wohltuender Klarheit. Sie zeigen ein interdisziplinäres Verständnis ihres Faches, verweigern sich biologistischer Einengung und Checklisten-Diagnostik, betonen die Bedeutung von verstehend-psychologischen Ansätzen und biographischer Analyse, berücksichtigen neuere Entwicklungen der Psychotherapie und scheuen sich nicht, problematische Tendenzen im forensischen Fachgebiet zu benennen. Resozialisierung und Gefahrenabwehr stehen in einem Spannungsverhältnis, das ihrem Verständnis nach nicht einseitig zuungunsten therapeutisch-rehabilitativer Bemühungen aufgelöst werden darf. Auch kritisieren sie die Regelungen des Therapieunterbringungsgesetzes zu Recht als Missbrauch der Psychiatrie. Mit vorurteilslosem Blick und respektvoller Haltung gegenüber dem Außenseiter mahnen sie die Gleichstellung psychisch kranker Straftäter mit nicht delinquenten psychisch Kranken an.

Erhellend ist ihre Konzeptualisierung „psychopathologischer Entwicklungen“ - süchtige, sexualpathologische und querulatorische Entwicklung -, die auf die Unterscheidung von Prozess und Entwicklung (K. Jaspers) rekurriert. Die Kategorie „psychopathologische Entwicklung" ist zwar nicht in der ICD-11 aufgeführt, repräsentiert aber eine forensisch relevante klinische Realität. Die Ausführungen der Autoren zur Gefahr einer "kochbuchartig" angewandten Affektkriteriologie verdienen besondere Beachtung. Auch ihr Hinweis auf definitorische "Unschärfen“ in den "Mindestanforderungen“ (2005) bezüglich der Schuldfähigkeitsbeurteilung Persönlichkeitsgestörter ist sehr ernst zu nehmen. Die von ihnen benannte Gefahr, dass eine restriktivere Anwendung genutzt werden kann, bereits Untergebrachte aus dem Maßregelvollzug „heraus zu definieren“, ist nicht von der Hand zu weisen. Das lesenswerte Kapitel zur forensisch-psychiatrischen Therapie berührt die Überleitung aus dem forensischen in das psychiatrische Regelsystem nur am Rande. Hier wünschte man sich für die nächste Auflage genauere Ausführungen, wie die Verzahnung beider Systeme verbessert werden kann.

Dem in steter Erweiterung befindlichen Werk ist inzwischen ein eigenes Kapitel zur Begutachtung in Sozialrecht und Versicherungsmedizin hinzugefügt worden. Der Band enthält zahlreiche illustrative Fallbeispiele und im Anhang zwei ausführliche Mustergutachten. Er regt zum kritischen Mitdenken an und sei jedem forensisch Interessierten, dem Einsteiger wie dem bereits Kundigen, wärmstens empfohlen. 